\title{
Risk Concentration and Diversification: Second-Order Properties
}

\author{
Matthias Degen ${ }^{\mathrm{a}, *}$, Dominik D. Lambrigger ${ }^{\mathrm{b}}$, Johan Segers $^{\mathrm{c}}$ \\ ${ }^{a}$ Department of Mathematics, ETH Zurich, 8092 Zurich, Switzerland \\ ${ }^{b}$ Department of Mathematics, ETH Zurich, 8092 Zurich, Switzerland \\ ${ }^{c}$ Institut de statistique, Université catholique de Louvain, B-1348 Louvain-la-Neuve, \\ Belgium
}

\begin{abstract}
The quantification of diversification benefits due to risk aggregation plays a prominent role in the (regulatory) capital management of large firms within the financial industry. However, the complexity of today's risk landscape makes a quantifiable reduction of risk concentration a challenging task. In the present paper we discuss some of the issues that may arise. The theory of second-order regular variation and second-order subexponentiality provides the ideal methodological framework to derive second-order approximations for the risk concentration and the diversification benefit.
\end{abstract}

Keywords: Diversification, second-order regular variation, second-order subexponentiality, subadditivity, Value-at-Risk

JEL Classification: C14

Subject Category and Insurance Brand Category: IE43

\section{Introduction}

Diversification is one of the most popular techniques to mitigate exposure to risk and constitutes an important part within the current regulatory framework for banks (Basel II) as well as in the preparation of the new regulatory framework for insurance companies (Solvency II). However, due to the increasing complexity of financial and insurance products, a quantitative anal-

\footnotetext{
*Corresponding author. Tel.: +41 446323428

Email address: degen@math.ethz.ch (Matthias Degen)
} 
ysis of diversification benefits has become a demanding task. Many authors have warned against an imprudent application of diversification concepts, especially when the underlying risk factors show a heavy-tailed pattern; see for instance Fama and Miller (1972), p. 269, Rootzén and Klüppelberg (1999), Embrechts et al. (2002) or Degen et al. (2007). More recently, Ibragimov and Walden (2007) and Ibragimov et al. (2009) have discussed diversification benefits linking heavy-tailed distributions to specific economic models.

In addition to that, the structure of internationally active financial groups has created the need to analyze diversification effects not only at the individual firm or subsidiary level, but also at the group level. For recent advances in the context of group supervision, see for instance Gatzert and Schmeiser (2008) or Gatzert et al. (2008) and references therein.

In the present paper we study mathematical properties of diversification effects under the risk measure Value-at-Risk (VaR). For a risky position $X$ with distribution function $F$, the Value-at-Risk at the level $\alpha$ is defined by $\operatorname{VaR}_{\alpha}(X)=F^{\leftarrow}(\alpha), 0<\alpha<1$, where $F^{\leftarrow}(\alpha)=\inf \{x \in \mathbb{R}: F(x) \geq \alpha\}$, denotes the generalized inverse of $F$. Under the Basel II/Solvency II framework, $\operatorname{VaR}_{\alpha}(X)$ essentially corresponds to the regulatory risk capital a financial institution needs to hold in order to be allowed to carry the risky position $X$ on its books. Note that the level $\alpha$ is given by the respective regulatory authority and is typically close to 1 .

Throughout we assume that our potential future losses $X_{1}, \ldots, X_{n}, n \geq$ 2 , are non-negative independent and identically distributed (iid) random variables with continuous distribution function $F$. We write $\bar{F}=1-F$ for the tail of $F$. We assume that $\bar{F} \in R V_{-1 / \xi}$, i.e. for every $x>0$,

$$
\frac{\bar{F}(t x)}{\bar{F}(t)} \rightarrow x^{-1 / \xi}, \quad t \rightarrow \infty
$$

so that

$$
C(\alpha)=\frac{\operatorname{VaR}_{\alpha}\left(\sum_{k=1}^{n} X_{k}\right)}{\sum_{k=1}^{n} \operatorname{VaR}_{\alpha}\left(X_{k}\right)} \rightarrow n^{\xi-1}, \quad \alpha \rightarrow 1 .
$$

We will refer to $C(\alpha)$ as the risk concentration (at level $\alpha$ ) and to $1-C(\alpha)$ as the diversification benefit.

For our mathematical discussion below, we do not restrict ourselves to a specific economic interpretation of the rvs $X_{k}$, but-with regard to possible applications - the reader might want to think of the following examples. Within the Basel II framework, the $X_{k}$ 's could for instance represent the 
yearly aggregated operational risk (OR) losses of a business line $k$ on an individual firm or subsidiary level; see Basel Committee on Banking Supervision (2006), $\S 657,669$. Or, in the context of group supervision, the $X_{k}$ 's might be viewed as the total yearly OR loss of a subsidiary company $k$; see Basel Committee on Banking Supe (2006), $\S 657$. In a Solvency II framework, one might think of the $X_{k}$ 's as the assets and/or liabilities of a subsidiary company $k$; see for instance Gatzert et al. (2008) for a discussion of risk concentration results in the nonheavy-tailed case.

Due to the non-coherence of VaR, diversification benefits may be positive or negative. A risk concentration value $C(\alpha)$ greater than 1 means nondiversification (i.e. superadditivity of $\operatorname{VaR}_{\alpha}$ ) at the level $\alpha$. In such cases, aggregation of risks would even lead to an increase of regulatory risk capital.

On a side note we remark that the issue of non-diversification does not arise for instance in the Swiss Solvency Test (SST), as there the coherent risk measure Expected Shortfall (ES) is used; see Artzner et al. (1999). Nevertheless, the asymptotic result (11) still holds under ES (provided that $\xi<1$ ).

Given the high $\alpha$-levels typically of interest for risk management practice, analyzing risk concentration by means of its empirical counterpart will in general not yield much insight. One is therefore advised to consider (suitable) analytic approximations of $C(\cdot)$. In the simple case of $n$ regularly varying iid losses, relation (1) gives rise to a first-order approximation $C_{1}(\alpha) \equiv n^{\xi-1}$ of $C(\alpha)$ for $\alpha$ close to 1 . The asymptotic result (11) has been generalized to situations where the vector $\mathbf{X}=\left(X_{1}, \ldots, X_{n}\right) \geq \mathbf{0}$ is multivariate regularly varying with index $-1 / \xi$ and with identically distributed margins; see Barbe et al. (2006), Proposition 2.1 and Embrechts et al. (2009), Theorem 4.1.

The main issue discussed in this paper is that the convergence in (1) may be arbitrarily slow. As a consequence, in risk management practice, where we are interested in $C(\alpha)$ at some fixed level of $\alpha$ typically ranging from $95 \%$ to $99.97 \%$, the first-order approximation $C_{1}(\alpha)$ may be too crude as the following example illustrates.

EXAMPLE 1.1 Consider a financial institution holding two risks $X_{1}$ and $X_{2}$. Assume that these positions are modeled by $X_{1}, X_{2} \stackrel{\text { iid }}{\sim} F$ with $\bar{F} \in R V_{-2}$. In that case the risk concentration satisfies $C(\alpha) \rightarrow 2^{\xi-1}=1 / \sqrt{2} \approx 0.71$, for $\alpha \rightarrow 1$. Therefore, when aggregating $X_{1}$ and $X_{2}$, a diversification benefit 
(reduction of regulatory risk capital) of about $29 \%$ would seem reasonable at first sight (for high levels of $\alpha$ ). Figure 1 however shows that such an argumentation needs careful rethinking.

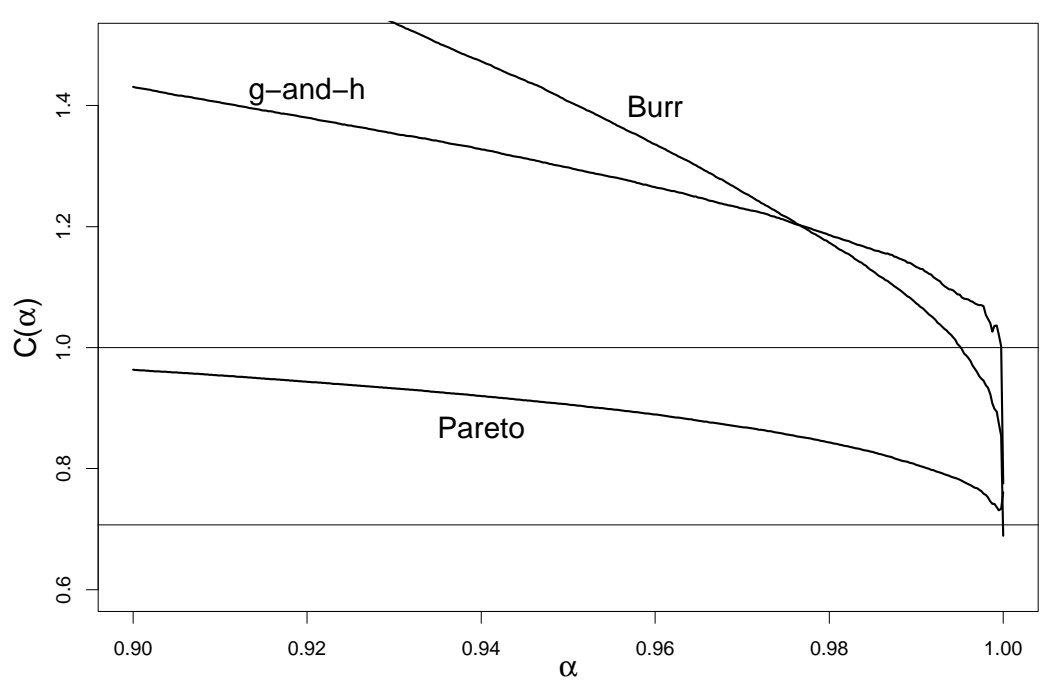

Figure 1: Empirical risk concentration (based on $10^{7}$ simulations) together with a first-order approximation $C_{1} \equiv 1 / \sqrt{2} \approx 0.71$ for two iid random variables from a $\operatorname{Burr}(\tau=0.25, \kappa=8)$, a Pareto $(\xi=0.5)$ and a g-and-h $(a=0, b=1, g=2, h=0.5)$ distribution; see Remark 2.3 and Examples 4.2 and 4.3 for the parameterization used in the respective models.

In what follows, we suggest the reader to keep Figure 1 in mind as a warning against a careless use of asymptotics to justify diversification benefits. Most importantly, the behavior of the risk concentration $C(\alpha)$ at levels of $\alpha$ close to 1 (typically of interest for applications) may be very sensitive, i.e. small changes of $\alpha$ may lead to large changes of $C(\alpha)$. In economic terms this means that while we may well expect diversification benefits of considerable size at a certain level $\alpha$, this may change rather drastically into non-diversification once we move away only little from that level.

Altogether this motivates the consideration of a second-order approxima- 
tion for the risk concentration $C$. Concerning methodology, we draw on the theories of second-order regular variation and second-order subexponentiality. Our main result, Theorem [3.1, gives the precise asymptotic behavior of the approximation error $C(\alpha)-n^{\xi-1}$ as $\alpha \rightarrow 1$. As it turns out, asymptotically two situations may arise. Without going into details at this point, it is either the asymptotic behavior in the second-order regular variation part or the one in the second-order subexponential part that dominates in the limit.

For the more applied risk management end-user, the main message is that, not only for infinite mean but also for finite mean models used in financial and insurance risk management, aggregating risks may somewhat surprisingly result in a negative diversification benefit (under VaR).

The paper is organized as follows. Section 2 recalls basic definitions and results on second-order regular variation and second-order subexponentiality. In Section 3 we present our main result on the second-order behavior of the risk concentration $C(\alpha)$. In Section 4 we apply this result to different distribution functions relevant for practice.

\section{Preliminaries}

The tail quantile function associated with the distribution function $F$ is denoted by $U_{F}(t)=(1 / \bar{F})^{\leftarrow}(t)=F^{\leftarrow}(1-1 / t), t>1$. Where clear from the context, we omit the subscript and write $U$ instead of $U_{F}$. Recall that $\bar{F} \in R V_{-1 / \xi}$ for some $\xi>0$ is equivalent to $U \in R V_{\xi}$. In this case we write $U(t)=t^{\xi} L_{U}(t)$, where $L_{U} \in R V_{0}$ denotes the slowly varying function associated with $U$.

The distribution function of the sum $X_{1}+\cdots+X_{n}$ is denoted by $G$ and due to the iid assumption we have $G(x)=F^{n *}(x)$, the $n$-fold convolution of $F$. Since $\bar{F}$ is regularly varying, $F$ is subexponential and hence

$$
\frac{\bar{G}(x)}{\bar{F}(x)} \rightarrow n, \quad x \rightarrow \infty
$$

see for instance Embrechts et al. (1997), Corollary 1.3.2. In terms of quantiles, setting $G^{\leftarrow}(\alpha)=x$, we obtain

$$
\frac{G^{\leftarrow}(\alpha)}{F^{\leftarrow}(\alpha)}=\frac{U_{F}(1 / \bar{F}(x))}{U_{F}(1 / \bar{G}(x))}=\frac{U_{F}(1 / \bar{F}(x))}{U_{F}(\underbrace{\bar{F}(x) / \bar{G}(x)}_{\rightarrow 1 / n} 1 / \bar{F}(x))} \rightarrow n^{\xi}, \quad \alpha \rightarrow 1,
$$


due to the Uniform Convergence Theorem for regularly varying functions; see for instance Bingham et al. (1987), Theorem 1.5.2. This implies

$$
C(\alpha)=\frac{\operatorname{VaR}_{\alpha}\left(\sum_{k=1}^{n} X_{k}\right)}{\sum_{k=1}^{n} \operatorname{VaR}_{\alpha}\left(X_{k}\right)} \rightarrow n^{\xi-1}, \quad \alpha \rightarrow 1 .
$$

In order to analyze the convergence rate of $C(\alpha)$ to $n^{\xi-1}$ as $\alpha \rightarrow 1$, the derivation in (2) suggests to study the second-order behavior in the two limit relations

$$
\begin{aligned}
& \frac{U(t s)}{U(t)} \rightarrow s^{\xi}, \quad t \rightarrow \infty, \\
& \bar{G}(x) \\
& \overline{\bar{F}(x)} \rightarrow n, \quad x \rightarrow \infty .
\end{aligned}
$$

Rate of convergence results for (3) are well-established within the framework of second-order regular variation; see for instance de Haan and Ferreira (2006), Section 2.3 and Appendix B.3 for an introduction. Rate of convergence results for (4) may be obtained using the framework of secondorder subexponentiality; see for instance Omey and Willekens (1986, 1987) and Barbe and McCormick (2005). Below we review these two concepts.

\subsection{Second-order regular variation}

Definition 2.1 (SECOnd-order Regular variation) A function $U \in$ $R V_{\xi}$ with $\xi>0$ is said to be of second-order regular variation with parameter $\rho \leq 0$, if there exists a function $a(\cdot)$ with $\lim _{t \rightarrow \infty} a(t)=0$ such that

$$
\lim _{t \rightarrow \infty} \frac{\frac{U(t s)}{U(t)}-s^{\xi}}{a(t)}=H_{\xi, \rho}(s)=s^{\xi} \frac{s^{\rho}-1}{\rho},
$$

with the obvious interpretation for $\rho=0$. In this case we write $U \in 2 R V_{\xi, \rho}(a)$ and refer to $a(\cdot)$ as the auxiliary function of $U$.

Note that $U \in 2 R V_{\xi, \rho}$ is equivalent to $\bar{F} \in 2 R V_{-1 / \xi, \rho / \xi}$ for $\xi>0, \rho \leq 0$. It is well known that if a non-trivial limit $H_{\xi, \rho}$ in (5) exists which is not a multiple of $s^{\xi}$, then it is necessarily of the form stated. Furthermore, the auxiliary function satisfies $|a| \in R V_{\rho}$; see for instance de Haan and Stadtmüller (1996), Theorem 1. The second-order parameter $\rho$ thus governs the rate of convergence in (3), i.e. the smaller $|\rho|$, the slower the convergence. 
A broad and frequently used subclass of models satisfying (5) is given by the so-called Hall class; see also Hall (1982).

Definition 2.2 (Hall Class) A distribution function $F$ is said to belong to the Hall class if its quantile function $U$ admits the asymptotic representation $U(t)=c t^{\xi}\left(1+d t^{\rho}+o\left(t^{\rho}\right)\right)$ as $t \rightarrow \infty$, for some $c>0, d \in \mathbb{R} \backslash\{0\}$, and first- and second-order parameters $\xi>0$ and $\rho<0$.

In terms of tail functions this means that we consider models of the form

$$
\bar{F}(x)=\left(\frac{x}{c}\right)^{-1 / \xi}\left(1+\frac{d}{\xi}\left(\frac{x}{c}\right)^{\rho / \xi}+o\left(x^{\rho / \xi}\right)\right), \quad x \rightarrow \infty .
$$

Note that the tail quantile function of a loss model in the Hall class obviously satisfies $U \in 2 R V_{\xi, \rho}(a)$ with $a(t) \sim d \rho t^{\rho}$ as $t \rightarrow \infty$. [Throughout the paper we mean by $f_{1}(t) \sim f_{2}(t)$ for $t \rightarrow t_{0}$ that $f_{1}(t) / f_{2}(t) \rightarrow 1$ as $t \rightarrow t_{0}$.] Conversely, loss models that are second-order regularly varying with $\rho<0$ and with auxiliary function $a(t) \sim d \rho t^{\rho}$ for $t \rightarrow \infty$ are members of the Hall class. This follows from the Representation Theorem for extended regularly varying functions; see Bingham et al. (1987), Theorem 3.6.6.

REMARK 2.3: For the standard Pareto model $U(t)=t^{\xi}$, the convergence in (3) is immediate. We interpret this case as $U \in 2 R V_{\xi,-\infty}$.

Heavy-tailed models $U \in 2 R V_{\xi, \rho}$ not belonging to the Hall class include for instance the loggamma or the g-and-h distribution ( $\rho=0$ in both cases).

\subsection{Second-order subexponentiality}

Second-order subexponentiality results by Barbe and McCormick (2005) (Theorems 2.2 and 2.5) are summarized in the following proposition; see also Omey and Willekens (1986, 1987) for similar results.

Proposition 2.4 Assume that $F$ is differentiable with $F(0)=0$. If for some $\xi>0, \bar{F}(x) /(x f(x)) \rightarrow \xi$ as $x \rightarrow \infty$, then for $n \geq 2$ and with $G(x)=F^{n *}(x)$,

$$
\lim _{x \rightarrow \infty} \frac{\overline{\bar{G}(x)}-n}{\overline{\bar{F}(x)}-n}=J_{\xi}(n)=n(n-1) c_{\xi}
$$


with

$$
c_{\xi}= \begin{cases}1 / \xi, & \text { if } \xi \leq 1 \\ (1-\xi) \frac{\Gamma^{2}(1-1 / \xi)}{2 \Gamma(1-2 / \xi)}, & \text { if } \xi>1\end{cases}
$$

and

$$
b(x)= \begin{cases}\mu_{F} / x, & \text { if } \xi \leq 1, \mu_{F}<\infty, \\ \mu_{F}(x) / x, & \text { if } \xi=1, \mu_{F}=\infty, \\ \bar{F}(x) /(\xi-1), & \text { if } \xi>1,\end{cases}
$$

where $\mu_{F}(x)=\int_{0}^{x} t d F(t), \mu_{F}=\lim _{x \rightarrow \infty} \mu_{F}(x)$ and where $\Gamma$ denotes the gamma function.

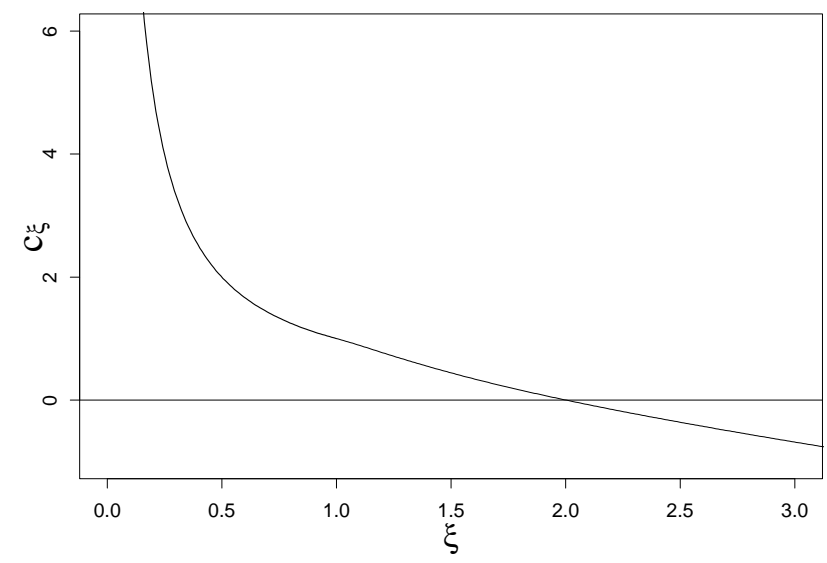

Figure 2: $c_{\xi}$ in Proposition 2.4 as a function of $\xi$.

\section{REMARKS:}

i) In defining $c_{\xi}$ for $1<\xi<2$ we make use of the analytic continuation of the gamma function $\Gamma$ to $\mathbb{C} \backslash\{0,-1,-2, \ldots\}$; see Abramowitz and Stegun (1972), Formula 6.1.2.

ii) Note that $c_{\xi}$ is strictly decreasing in $\xi$ and thus $c_{\xi}=0$ if and only if $\xi=2$; see also Figure 2, In that case, Proposition 2.4 does not yield a (proper) second-order result for convolutions in that particular case. 
To the best of our knowledge, second-order asymptotics of the above form for $\xi=2$ are not available in the literature (except for special cases, such as stable laws).

iii) In the case $\xi=1$ and $\mu_{F}=\infty$, we have $f=F^{\prime} \in R V_{-2}$. Karamata's Theorem implies that $\mu_{F}(x)=\int_{0}^{x} t f(t) d t$ is slowly varying; see for example de Haan and Ferreira (2006), Theorem B.1.5.

iv) By Proposition 2.4, the asymptotic behavior of the function $b$ is fully specified and we have $b \in R V_{-(1 \wedge 1 / \xi)}$ with $\xi>0$.

\section{Main result}

Combining the concepts of second-order regular variation and secondorder subexponentiality, we obtain a second-order result for the risk concentration $C$. It may be viewed as a partial quantile analogue of Theorem 3.2 in Geluk et al. (1997). Recall the notations in the previous sections, in particular in Proposition 2.4.

\section{Theorem 3.1}

Let $X_{1}, \ldots, X_{n} \stackrel{i \text { id }}{\sim} F$ be positive random variables and let $U=(1 / \bar{F})^{\leftarrow}$ be such that $t U^{\prime}(t) / U(t) \rightarrow \xi>0$. Suppose that $U \in 2 R V_{\xi, \rho}(a)$ for some $\rho \leq 0$ and with auxiliary function $a(\cdot)$ of ultimately constant sign. If $\rho \neq-(1 \wedge \xi)$, then, for fixed $n \geq 2$ and as $\alpha \rightarrow 1$,

$$
C(\alpha)=\frac{\operatorname{VaR}_{\alpha}\left(\sum_{k=1}^{n} X_{k}\right)}{\sum_{k=1}^{n} \operatorname{VaR}_{\alpha}\left(X_{k}\right)}=n^{\xi-1}+K_{\xi, \rho}(n) A(\alpha)+o(A(\alpha))
$$

where $A(\alpha)$ and $K_{\xi, \rho}(n)$ are given as follows:

(i) case $\rho<-(1 \wedge \xi)$ :

$$
\begin{gathered}
A(\alpha)=b\left(F^{\leftarrow}(\alpha)\right)= \begin{cases}\mu_{F} / F^{\leftarrow}(\alpha), & \text { if } \xi \leq 1, \rho<-\xi, \mu_{F}<\infty, \\
\mu_{F}\left(F^{\leftarrow}(\alpha)\right) / F^{\leftarrow}(\alpha), & \text { if } \xi=1, \rho<-1, \mu_{F}=\infty, \\
(1-\alpha) /(\xi-1), & \text { if } \xi>1, \rho<-1 ;\end{cases} \\
K_{\xi, \rho}(n)= \begin{cases}(n-1) / n, & \text { if } \xi \leq 1, \rho<-\xi, \\
n^{\xi-2}(n-1) \xi c_{\xi}, & \text { if } \xi>1, \rho<-1 ;\end{cases}
\end{gathered}
$$


(ii) case $\rho>-(1 \wedge \xi)$ :

$$
\begin{aligned}
A(\alpha) & =a(1 /(1-\alpha)), \\
K_{\xi, \rho}(n) & =n^{\xi-1} \frac{n^{\rho}-1}{\rho} .
\end{aligned}
$$

Proof: See Appendix.

As an approximation to the risk concentration $C(\alpha)$, Theorem 3.1 suggests to consider a second-order approximation $C_{2}(\alpha)=n^{\xi-1}+K_{\xi, \rho}(n) A(\alpha)$, for $\alpha<1$.

According to Theorem 3.1 two situations arise. Note that

$$
A(\alpha)= \begin{cases}b\left(F^{\leftarrow}(\alpha)\right), & \text { if } \rho<-(1 \wedge \xi), \\ a(1 /(1-\alpha)), & \text { if } \rho>-(1 \wedge \xi),\end{cases}
$$

where $b \circ F^{\leftarrow} \in R V_{-(1 \wedge \xi)}$ and $|a| \in R V_{\rho}$. Now if $\rho<-(1 \wedge \xi)$, then $b\left(F^{\leftarrow}(\alpha)\right)$ vanishes faster than $a(1 /(1-\alpha))$ as $\alpha \rightarrow 1$. This motivates the following terminology: a loss model is said to be of fast convergence if $U \in 2 R V_{\xi, \rho}$ with first- and second-order parameters satisfying $\rho<-(1 \wedge \xi)$, and of slow convergence, if $\rho>-(1 \wedge \xi)$; see Figure 3 .

A refinement of the proof of Theorem 3.1 also allows to treat the boundary case $\rho=-(1 \wedge \xi)$. This case seems to be of less relevance for practical applications though.

\section{Addendum}

Let $U \in 2 R V_{\xi, \rho}(a)$ satisfy the conditions of Theorem 3.1. Suppose, in addition, that for some $q \in \mathbb{R} \backslash\{0\}$

$$
\frac{b\left(F^{\leftarrow}(\alpha)\right)}{a\left(\frac{1}{1-\alpha}\right)} \rightarrow q, \quad \alpha \rightarrow 1,
$$

where $b(\cdot)$ is as in Proposition 2.4. Then, for $n \geq 2$ and $\alpha \rightarrow 1$,

$C(\alpha)=n^{\xi-1}+\left(\xi n^{\xi-2} n^{-(1 \wedge \xi)} J_{\xi}(n) q+n^{-1} H_{\xi, \rho}(n)\right) a\left(\frac{1}{1-\alpha}\right)+o\left(a\left(\frac{1}{1-\alpha}\right)\right)$,

with $H_{\xi, \rho}(\cdot)$ and $J_{\xi}(\cdot)$ as in Definition 2.1 and Proposition 2.4 respectively. 


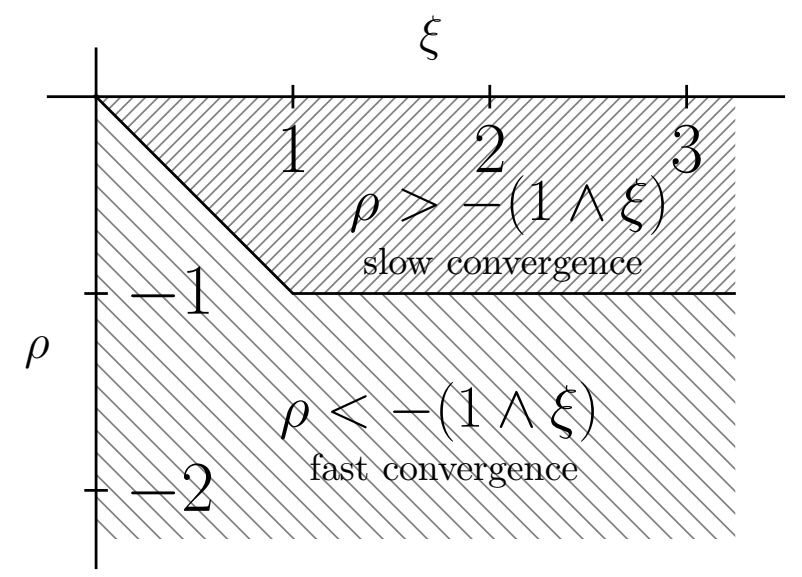

Figure 3: Illustration of the three possible cases, $\rho>-(1 \wedge \xi)$ (fast convergence), $\rho<-(1 \wedge \xi)$ (slow convergence) and $\rho=$ $-(1 \wedge \xi)$ (boundary case) in the $(\xi, \rho)$-parameter space for loss models $U \in 2 R V_{\xi, \rho}$.

REMARK 3.2: Assume that $U \in 2 R V_{\xi, \rho}(a)$. If the associated slowly varying function $L_{U}$ is differentiable with ultimately monotone derivative $L_{U}^{\prime}$, then the auxiliary function $a(\cdot)$ can be chosen as

$$
a(t)=\frac{t U^{\prime}(t)}{U(t)}-\xi
$$

A proof is given in the Appendix.

\section{Examples}

In this section we consider the situation of Theorem 3.1 for different loss models. For notational convenience we focus on the case $\rho \neq-(1 \wedge \xi)$. For the Hall class, Theorem 3.1 specializes as follows.

Corollary 4.1 Let $U$ belong to the Hall class (i.e. $U(t)=c t^{\xi}\left(1+d t^{\rho}+\right.$ $\left.o\left(t^{\rho}\right)\right)$ as $t \rightarrow \infty$, for some $c>0, d \in \mathbb{R} \backslash\{0\}, \xi>0$, and $\left.\rho<0\right)$ and satisfy the assumptions of Theorem 3.1. Then, the function $A(\cdot)$ in Theorem 3.1 can be chosen as

$$
A(\alpha)= \begin{cases}\frac{\mu_{F}}{c}(1-\alpha)^{\xi}, & \text { if } \xi \leq 1, \rho<-\xi, \mu_{F}<\infty, \\ -(1-\alpha) \log (1-\alpha), & \text { if } \xi=1, \rho<-1, \mu_{F}=\infty, \\ (1-\alpha) /(\xi-1), & \text { if } \xi>1, \rho<-1, \\ d \rho(1-\alpha)^{-\rho}, & \text { if } \rho>-(1 \wedge \xi) .\end{cases}
$$


ExAmple 4.2 (Burr) Let $X_{1}, \ldots, X_{n} \stackrel{\text { iid }}{\sim} \operatorname{Burr}(\tau, \kappa)$, with tail function $\bar{F}(x)=\left(1+x^{\tau}\right)^{-\kappa}$ for some $\tau, \kappa>0$. In terms of its tail quantile function this writes as

$$
U(t)=\left(t^{1 / \kappa}-1\right)^{1 / \tau}=t^{1 /(\tau \kappa)}\left(1-\frac{1}{\tau} t^{-1 / \kappa}+o\left(t^{-1 / \kappa}\right)\right), \quad t \rightarrow \infty,
$$

so that $U$ belongs to the Hall class with parameters $c=1, d=-1 / \tau$, $\xi=1 /(\tau \kappa)$, and $\rho=-1 / \kappa$. By (마) the tail quantile function $U$ is given in an explicit form as well as through an asymptotic expansion, so that we may use either Theorem 3.1 or Corollary 4.1 to derive a second-order result for $C$. Using the former we obtain in the case of fast convergence, i.e. for $\kappa \wedge(1 / \tau)<1$

$$
C(\alpha)= \begin{cases}n^{1 /(\tau \kappa)-1}+\left(\frac{n-1}{n} \kappa B\left(\kappa-\frac{1}{\tau}, 1+\frac{1}{\tau}\right)+o(1)\right)(1-\alpha)^{1 /(\tau \kappa)}, & \text { if } \tau \kappa>1, \\ 1-\left(\frac{n-1}{n}+o(1)\right)(1-\alpha) \log (1-\alpha), & \text { if } \tau \kappa=1, \\ n^{1 /(\tau \kappa)-1}-\frac{n-1}{\tau \kappa} n^{1 /(\tau \kappa)-2} \frac{\Gamma^{2}(1-\tau \kappa)}{2 \Gamma(1-2 \tau \kappa)}(1-\alpha)+o(1-\alpha), & \text { if } \tau \kappa<1,\end{cases}
$$

as $\alpha \rightarrow 1$ and where $B(x, y)=\frac{\Gamma(x) \Gamma(y)}{\Gamma(x+y)}$ denotes the Beta function.

In case of slow convergence, i.e. if $\kappa \wedge(1 / \tau)>1$, Theorem 3.1 together with Remark 3.2 suggests to consider the expansion

$$
C(\alpha)=n^{1 /(\tau \kappa)-1}+\frac{1}{\tau} n^{1 /(\tau \kappa)-1}\left(1-n^{-1 / \kappa}\right)(1-\alpha)^{1 / \kappa}+o\left((1-\alpha)^{1 / \kappa}\right), \quad \alpha \rightarrow 1 .
$$

The gain of a second-order approximation $C_{2}$ over a first-order approximation $C_{1}$ is illustrated in Figure 4 for the case of a fast converging Burr and an exact Pareto model.

For practical purposes, it is essential to know whether $C(\alpha)$ approaches its ultimate value $n^{\xi-1}$ from above or from below as $\alpha$ tends to 1 . For a loss model $U \in 2 R V_{\xi, \rho}$ satisfying the assumptions of Theorem 3.1 with $\rho<-(1 \wedge \xi)$ (fast convergence case), the derivative of $C_{2}$ satisfies

$$
\lim _{\alpha \rightarrow 1} C_{2}^{\prime}(\alpha)= \begin{cases}-\infty, & \text { if } \xi<1, \text { or } \xi=1, \mu_{F}=\infty \\ -\frac{n-1}{n} \frac{\mu_{F}}{c}, & \text { if } \xi=1, \mu_{F}<\infty \\ n^{\xi-2}(n-1) \xi \frac{\Gamma^{2}(1-1 / \xi)}{2 \Gamma(1-2 / \xi)}, & \text { if } \xi>1\end{cases}
$$



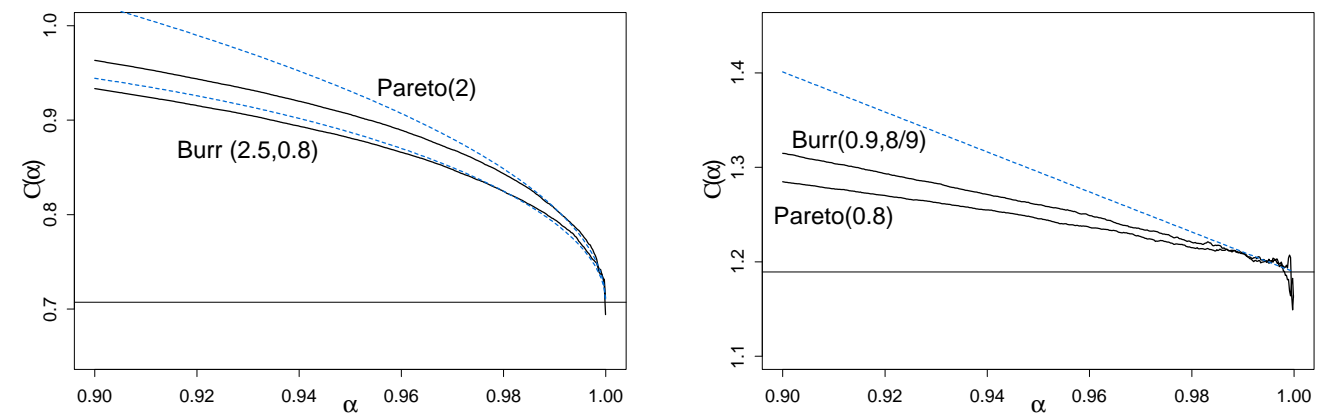

Figure 4: Empirical risk concentration (full, based on $10^{7}$ simulations) together with first-order approximation $C_{1} \equiv 1 / \sqrt{2} \approx 0.71$ (left panel) and $C_{1} \equiv 2^{1 / 4} \approx 1.19$ (right panel) and second-order approximation $C_{2}$ (dashed) for two iid $\operatorname{Burr}(\tau, \kappa)$ and $\operatorname{Pareto}(1 / \xi)$ random variables for a finite mean case (left panel) and an infinite mean case (right panel). Note the different scales on the vertical axis.

for some $c=\lim _{t \rightarrow \infty} L_{U}(t) \in(0, \infty)$. Moreover, we have $C_{2}^{\prime}(1)>0$ if and only if $\xi>2$, i.e. it is only in very heavy-tailed cases that $C(\alpha)$ increases to $n^{\xi-1}$ as $\alpha \rightarrow 1$.

In the case of slowly converging loss models, the situation is more involved. For $U \in 2 R V_{\xi, \rho}$ with $\rho>-(1 \wedge \xi)$, the ultimate behavior of $C_{2}^{\prime}$ depends on the exact form of $L_{U}$ so that in general no precise statement can be made without extra assumptions. Still, for distributions in the Hall class, $C(\alpha)$ will approach its limit $n^{\xi-1}$ from above (below) if $d$ is negative (positive).

Within the class of slowly converging loss models, the case $\rho=0$ deserves special attention, as in this case the decay of $|a|$ in (5) may be arbitrarily slow. This is due to the behavior of the associated slowly varying function $L_{U}$ which, in the case $\rho=0$, may indeed be rather misleading; see for instance Degen and Embrechts (2008), Section 4. A prime example for this is provided for instance by Tukey's g-and-h distribution.

ExAmPle 4.3 (g-and-h) A random variable $X$ is said to follow Tukey's g-and-h distribution with parameters $a, b, g, h \in \mathbb{R}$, if $X$ satisfies

$$
X=a+b \frac{e^{g Z}-1}{g} e^{h Z^{2} / 2},
$$


where $Z \sim \mathcal{N}(0,1)$ and with the obvious interpretation for $g=0$. Note that in principle such random variables need not be positive. In for financial risk management practice relevant cases the parameters typically satisfy $b, g, h>$ 0 , so that we may bypass this issue using the notion of right tail dominance; see Barbe and McCormick (2005).

Suppose $X_{1}, \ldots, X_{n}$ are iid g-and-h random variables with $a=0, b=1$ and $g, h>0$. Then one shows that $U \in 2 R V_{\xi, \rho}$ with $\xi=h, \rho=0$ and that

$$
\begin{aligned}
a\left(\frac{1}{1-\alpha}\right) & =\frac{U^{\prime}\left(\frac{1}{1-\alpha}\right)}{(1-\alpha) U\left(\frac{1}{1-\alpha}\right)}-\xi \\
& =\frac{g}{\Phi^{-1}(\alpha)}+O\left(\frac{1}{\left(\Phi^{-1}(\alpha)\right)^{2}}\right), \quad \alpha \rightarrow 1
\end{aligned}
$$

where we used the standard asymptotic expansion for the tail of the normal distribution given by $\bar{\Phi}(x)=e^{-x^{2} / 2} /(\sqrt{2 \pi} x)\left(1+O\left(1 / x^{2}\right)\right), x \rightarrow \infty$; see for instance Abramowitz and Stegun (1972), p. 932. Therefore, we obtain the following second-order asymptotics for the risk concentration:

$$
C(\alpha)=n^{h-1}+n^{h-1} \log (n) \frac{g}{\Phi^{-1}(\alpha)}+o\left(\frac{1}{\Phi^{-1}(\alpha)}\right), \quad \alpha \rightarrow 1 .
$$

Depending on the parameter values of $g$ and $h, C(\cdot)$ may be growing extremely fast when moving away from $\alpha=1$; see Figure 5. In that figure we compare a g-and-h with a Burr model of slow convergence and with a standard Pareto model. Note that we choose the tail index as $\xi=0.5$ in each model.

Figure 5 allows us to draw the following conclusions. Even at high levels of $\alpha<1$, the diversification benefit promised by first-order theory may vanish rather quickly and may even get negative. For the g-and-h model of Figure 5. the regime switch from sub- to superadditivity takes place at the extreme level of $\alpha \approx 99.95 \%$. The second-order approximation $C_{2}$ is able to capture this behavior better than $C_{1}$.

\section{Appendix}

Proof (Theorem 3.1): For $\alpha<1$, define $x=G^{\leftarrow}(\alpha)=\left(F^{n *}\right)^{\leftarrow}(\alpha)$. Note that the convergence in the definition of second-order regular variation holds locally uniformly on $(0, \infty)$; see de Haan and Ferreira (2006), Remark 


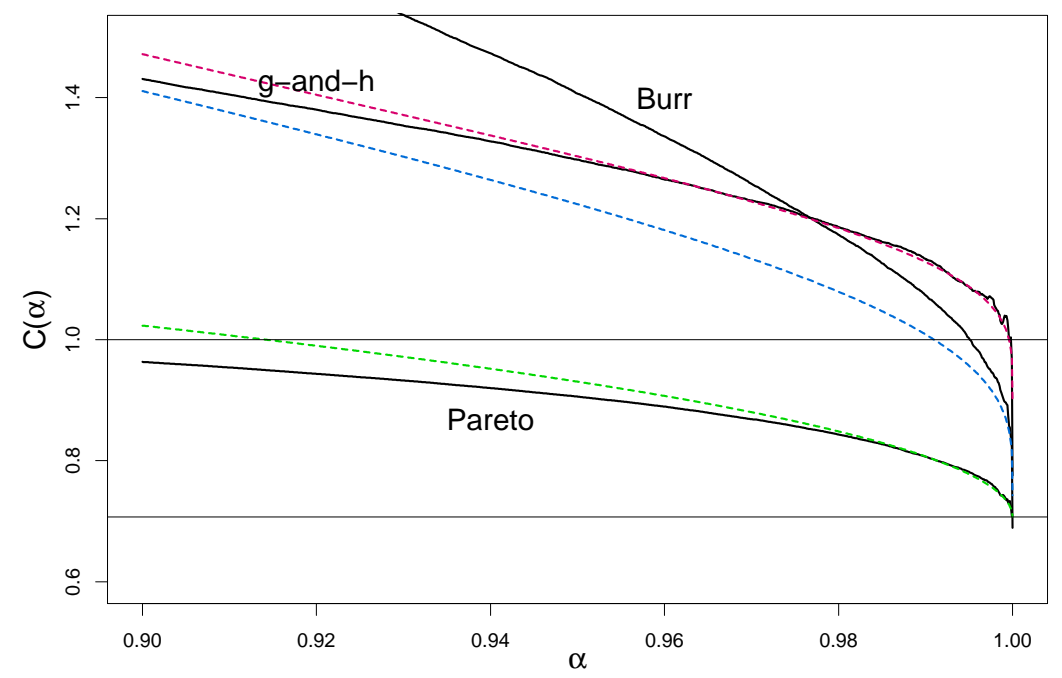

Figure 5: Empirical risk concentration (full, based on $n=10^{7}$ simulations) together with the first-order approximation $C_{1}(\alpha) \equiv 1 / \sqrt{2} \approx 0.71$ and the second-order approximation $C_{2}$ (dashed) for two iid Burr $(\tau=0.25, \kappa=8)$, Pareto $(\xi=0.5)$ and g-and-h $(g=2, h=0.5)$ random variables.

B.3.8. Therefore, replacing $t$ by $1 / \bar{G}(x)$ and $s$ by $\bar{G}(x) / \bar{F}(x)$ in (15), $U \in$ $2 R V_{\xi, \rho}$ implies

$$
\lim _{x \rightarrow \infty} \frac{\frac{U(1 / \bar{F}(x))}{n U(1 / \bar{G}(x))}-\frac{1}{n}\left(\frac{\bar{G}(x)}{\bar{F}(x)}\right)^{\xi}}{a(1 / \bar{G}(x))}=n^{\xi-1} \frac{n^{\rho}-1}{\rho}=n^{-1} H_{\xi, \rho}(n),
$$

with the obvious interpretation for $\rho=0$. From Proposition 2.4 we then get

$$
\begin{aligned}
C(\alpha)= & \frac{1}{n}\left(\frac{\bar{G}(x)}{\bar{F}(x)}\right)^{\xi}+n^{-1} H_{\xi, \rho}(n) a\left(\frac{1}{1-\alpha}\right)+o\left(a\left(\frac{1}{1-\alpha}\right)\right) \\
= & n^{\xi-1}\left(1+\xi n^{-1} J_{\xi}(n) b\left(G^{\leftarrow}(\alpha)\right)\right)+n^{-1} H_{\xi, \rho}(n) a\left(\frac{1}{1-\alpha}\right) \\
& +o\left(b\left(G^{\leftarrow}(\alpha)\right)\right)+o\left(a\left(\frac{1}{1-\alpha}\right)\right),
\end{aligned}
$$


for $\alpha \rightarrow 1$ and where we have used the expansion $(1+y)^{\xi}=1+\xi y+o(y)$ as $y \rightarrow 0$. Note that, due to regular variation of $b$, we have

$$
\frac{b\left(G^{\leftarrow}(\alpha)\right)}{b\left(F^{\leftarrow}(\alpha)\right)} \sim\left(\frac{G^{\leftarrow}(\alpha)}{F^{\leftarrow}(\alpha)}\right)^{-(1 \wedge 1 / \xi)} \rightarrow n^{-(\xi \wedge 1)}, \quad \alpha \rightarrow 1 .
$$

Define $\widetilde{A}(\alpha)=b\left(F^{\leftarrow}(\alpha)\right)+a\left(\frac{1}{1-\alpha}\right)$. Note that $b \circ F^{\leftarrow} \in R V_{-(1 \wedge \xi)}$ and $|a| \in$ $R V_{\rho}$. Due to the regular variation properties of $b$ and $a$ and since $\rho \neq-(1 \wedge \xi)$ this implies that

$$
\frac{C(\alpha)-n^{\xi-1}}{\widetilde{A}(\alpha)}=\xi n^{\xi-2} n^{-(\xi \wedge 1)} J_{\xi}(n) \frac{b\left(F^{\leftarrow}(\alpha)\right)}{\widetilde{A}(\alpha)}+n^{-1} H_{\xi, \rho}(n) \frac{a\left(\frac{1}{1-\alpha}\right)}{\widetilde{A}(\alpha)}+o(1),
$$

as $\alpha \rightarrow 1$, which yields the result.

Proof (Remark 3.2): $\quad U \in 2 R V_{\xi, \rho}(a)$ (with $\rho \leq 0<\xi$ ) with auxiliary function $a(\cdot)$ implies $U \in R V_{\xi}$ and we write $U(t)=t^{\xi} L(t)$ for some slowly varying function $L$. With this notation and for $s>0$,

$$
\lim _{t \rightarrow \infty} \frac{\frac{U(t s)}{U(t)}-s^{\xi}}{a(t)}=s^{\xi} \frac{s^{\rho}-1}{\rho} \Longleftrightarrow \lim _{t \rightarrow \infty} \frac{L(t s)-L(t)}{a(t) L(t)}=\frac{s^{\rho}-1}{\rho} .
$$

Hence $L \in E R V_{\rho}(B)$, i.e. $L$ is extended regularly varying with index $\rho \leq 0$ and auxiliary function $B(t)=a(t) L(t)$. For an introduction to ERV we refer to de Haan and Ferreira (2006), Appendix B.2.

Case $\rho=0$ : We write $L(t)=L\left(t_{0}\right)+\int_{t_{0}}^{t} L^{\prime}(s) d s$. The ultimate monotonicity of $L^{\prime}$ guarantees $L^{\prime} \in R V_{-1}$ by the Monotone Density Theorem for $\Pi$-variation; see Bingham et al. (1987), Corollary 3.6.9. In that case, $t L^{\prime}(t)$ is an auxiliary function, hence necessarily $B(t) \sim t L^{\prime}(t), t \rightarrow \infty$; see de Haan and Ferreira (2006), Remark B.2.6.

Case $\rho<0$ : In that case, the $\operatorname{limit}_{t \rightarrow \infty} L(t)=L(\infty)$ exists and is finite. Set $f(t)=L(\infty)-L(t)=\int_{t}^{\infty} L^{\prime}(s) d s$. Then $\lim _{t \rightarrow \infty} \frac{f(t)}{B(t)}=-1 / \rho$ and $f(t) \in R V_{\rho}$, by Theorem B.2.2 in de Haan and Ferreira (2006). Ultimate monotonicity of $L^{\prime}$ implies that $\frac{t L^{\prime}(t)}{f(t)} \rightarrow-\rho$ by Proposition B.1.9 11) of de Haan and Ferreira (2006) and hence $B(t) \sim t L^{\prime}(t)$ as $t \rightarrow \infty$.

Altogether, we thus obtain $a(t)=\frac{B(t)}{L(t)} \sim \frac{t L^{\prime}(t)}{L(t)}=\frac{t U^{\prime}(t)}{U(t)}-\xi$ as $t \rightarrow \infty$. 


\section{Acknowledgments}

The authors would like to thank Paul Embrechts and Mario V. Wüthrich for several discussions related to this paper and the numerous useful comments as well as the referee for constructive suggestions. The first two authors gratefully acknowledge the financial support by RiskLab Switzerland. The research of the third author is supported by IAP research network grant nr. P6/03 of the Belgian government (Belgian Science Policy) and by contract nr. 07/12/002 of the Projet d'Actions de Recherche Concertées of the

Communauté française de Belgique, granted by the Académie universitaire Louvain.

Abramowitz, M., Stegun, I. A., 1972. Handbook of Mathematical Functions. Dover Publications, New York.

Artzner, P., Delbaen, F., Eber, J. M., Heath, D., 1999. Coherent measures of risk. Mathematical Finance 9, 203-228.

Barbe, P., Fougères, A.-L., Genest, C., 2006. On the tail behavior of sums of dependent risks. Astin Bulletin 36, 361-373.

Barbe, P., McCormick, W. P., 2005. Asymptotic expansions of convolutions of regularly varying distributions. Journal of the Australian Mathematical Society 78, 339-371.

Bingham, N. H., Goldie, C. M., Teugels, J. L., 1987. Regular Variation. Cambridge University Press, Cambridge.

de Haan, L., Ferreira, A., 2006. Extreme Value Theory: An Introduction. Springer, New York.

de Haan, L., Stadtmüller, U., 1996. Generalized regular variation of second order. Journal of the Australian Mathematical Society 61, 381-395.

Degen, M., Embrechts, P., 2008. EVT-based estimation of risk capital and convergence of high quantiles. Advances in Applied Probability 40, 696715 .

Degen, M., Embrechts, P., Lambrigger, D. D., 2007. The quantitative modeling of operational risk: between g-and-h and EVT. Astin Bulletin 37, 265-291. 
Embrechts, P., Klüppelberg, C., Mikosch, T., 1997. Modelling Extremal Events for Insurance and Finance. Springer, Berlin.

Embrechts, P., Lambrigger, D. D., Wüthrich, M. V., 2009. Multivariate extremes and the aggregation of dependent risks: Examples and counterexamples. Extremes 12, 107-127.

Embrechts, P., McNeil, A. J., Straumann, D., 2002. Correlation and dependence in risk management: properties and pitfalls. In: Risk management: Value at Risk and Beyond. Ed. M. Dempster. Cambridge University Press, Cambridge, 176-223.

Fama, E. T., Miller, M. H., 1972. The Theory of Finance. Dryden Press, Hinsdale.

Gatzert, N., Schmeiser, H., 2008. On the risk situation of financial conglomerates: does diversification matter. Working Paper, University of St. Gallen.

Gatzert, N., Schmeiser, H., Schuckmann, S., 2008. Enterprise risk management in financial groups: Analysis of risk concentration and default risk. Financial Markets and Portfolio Management 22, 241-258.

Geluk, J., de Haan, L., Resnick, S., Stărică, C., 1997. Second-order regular variation, convolutions and the central limit theorem. Stochastic Processes and their Applications 69, 139-159.

Hall, P., 1982. On some simple estimates of an exponent of regular variation. Journal of the Royal Statistical Society Series B 44, 37-42.

Ibragimov, R., Jaffee, D., Walden, J., 2009. Nondiversification traps in catastrophe insurance markets. Review of Financial Studies 22, 959-993.

Ibragimov, R., Walden, J., 2007. The limits of diversification when losses may be large. Journal of Banking and Finance 31, 2551-2569.

Omey, E., Willekens, E., 1986. Second order behaviour of the tail of a subordinated probability distribution. Stochastic Processes and their Applications 21, 339-353.

Omey, E., Willekens, E., 1987. Second-order behaviour of distributions subordinate to a distribution with finite mean. Communications in Statistics: Stochastic Models 3, 311-342. 
Rootzén, H., Klüppelberg, C., 1999. A single number can’t hedge against economic catastrophes. Ambio 28, 550-555.

Basel Committee on Banking Supervision, 2006. International convergence of capital measurement and capital standards - a revised framework. Basel: Bank for International Settlements. 